\title{
Ethical issues of diagnosis and therapy in patients with HIV-AIDS and Tuberculosis
}

${ }^{*}$ Clinic of Infectious Diseases, Faculty of Medicine, "Ovidius" University Constanta

${ }^{* *}$ Clinic of Pulmonology, Faculty of Medicine, "Ovidius" University Constanta

\begin{abstract}
.
Like medicine, ethics is a practical discipline, which they acquire the knowledge of the general principles and skills to solve problems. Long term care involves both clinical and ethical issues in medicine. Effective medical education of long-suffering patients has to be focusing on increasing their adherence to treatment. The occurrence of AIDS pandemic has changed the physician's way of thinking in terms of ethics in general, and in medicine in particular. HIV/AIDS has focused attention on deficiencies in the field of therapy, long care issues, medical training and education of the patients, as well as in deontology. The treatment of HIV infected patients with all new drugs discovered is not yet completely curative and the association of opportunistic infections, as tuberculosis (TB), is representing a permanent challenge for therapist.

Both ethical and medical training in general as regards AIDS and TB, should focus mainly on exposed people and learning through experience, to form skills that would make doctors to work with sensitivity in regard to sexuality, substance abuse, lack of treatment, relapses of disease and even death. In the same time, any ethical or cultural environment can enrich and support the real moral education of medical staff.

Medical ethics and ethics relating specifically to TB
\end{abstract}

Arghir Oana Cristina

Clinic of Pulmonology, Faculty of Medicine, "Ovidius" University Constanta

Str. Sentinelei nr. 40, cod. 900002 Constanta

Tel: +40722636201

e-mail: arghir_oana@yahoo.com and HIV/AIDS, often meet highly complex problems. Alternative possibilities and decisions to take of what is right or wrong may create a real ethical, clinical and therapeutic dilemma.

Keywords: HIV/AIDS; tuberculosis; medical ethics; therapy; prevention

\section{Introduction}

Ethics in health today is based on the most often conceptual framework which is used in analyzing the four "principles of bio-ethics". These fundamental principles consist in: respect for individual autonomy, benefits, not harm, justice. Currently, there is no way to choose the principles when practice comes into conflict with theory. Theories and all 4 fundamental principles of ethics alone cannot solve both medical and individual problems in patients infected with HIV-AIDS, because there may be mutually exclusive decisions that can violate certain principles while others are supported. This is the so called ethical dilemma [1,2]. Physicians need to spend a lot of times in the face of questions: whether or not to violate some principle or theory. There are possible alternatives but they may violate the rights of the patient more or less. The ethical principles by themselves are not 
sufficient to reach a conclusion in such a case. An ethical dimension may appear when it is necessary to find the most appropriate international scientific vocabulary and a common language to discuss the medico-moral problems with the patient [3].

Most medical staff working in sections of sexually transmitted diseases or infectious diseases or in communities with high knowledge of HIV/AIDS and TB experience in providing clinical services, training programs and can become important qualified transmitters of information.

The changes created by AIDS in patients with related TB require the use of new approaches to teaching ethics in medicine: ethics of relations between medical staff and patients as well as interprofessionals relations, and ethics in launching health policies and planning against TB and/or HIV.

There are a multitude of targets in relation to HIV infection and TB, more complex than ever, which have a great implication in teaching ethics in medicine:

- Attitude toward sexuality, sex education, and protected sex;

- Respect the dignity and human rights against people with AIDS;

- Policies promoting non-discrimination against people with AIDS;

- Adoption of non-coercive approach towards public health and screening;

- Encouraging economic development in poverty reduction and ignorance;

- Liquidation of social inequality and women subordination, which put them at risk of AIDS;

- Co-infection HIV - TB;

- Treatment of tuberculosis in the setting of HIV;

- Drug interactions and intolerances;

- Issues specifically regarding multidrugresistant TB.

Regarding relations between medical workers and patients, traditional medical ethics was based on the paradigm of therapy intervention in case of crisis, where duty of medical personnel is to provide care (protection) and the mean to cure. This model is used especially in emergency assistance or in medical care of children. Adults, the sexually active ones with HIV/AIDS, many of those who were educated, may demand their rights. Population HIV related
TB groups must be involved in projects for fighting against any form of suppression, discrimination and marginalization and lack of respect for their dignity. Health care workers must facilitate an appropriate implementation of guidelines, surveillance of the patients and management of therapy. Regarding HIV infection and TB, should be more democratic forms of approach in the medical profession, to work with teams of nurses, social workers and community.

\section{Ethical judgments applied in HIV / AIDS}

Medical and moral problems raised by HIV/ AIDS with or without related TB are often complex and ambiguous, with no decisive arguments of either party. However, a decision is frequently a practical urgency. It is necessary to differentiate between guidelines recommendations, medical technology and/or scientific information, and alternative solutions to medical factors. Researchers consider that there is even a Christian ethic and they recommend to health workers to translate the medical and scientific knowledge into a language understood by all people informed in good faith and translated into action full of meaning [4].

\section{Confidentiality}

Confidentiality means that information that a person wishes to keep for her or a person who trusts (a doctor or counselor) will remain secret and not discussed by anyone outside the relationship of mutual trust, which is both protected by specific obligations. Confidentiality of personal health information is required both by respect for autonomy and by traditional medical ethics. Lack of privacy inhibits responsible decision making. This feature is important in sensitive information such as HIV positive status of a person, modes of HIV infection and TB co-infection, symptoms of HIV/AIDS and TB, natural evolution of both infections, and prognosis under therapy.

By maintaining confidentiality and trust, doctors and counselors are in a unique position to influence behavior, thus reducing the risk of transmitting HIV to others but have no capacity of preventing the evolution of TB infection to an active disease. If these skills in communication and mutual trust are well developed, the doctor-patient relationship 
can become an opportunity to change things for the better. On the other hand, the lack of respect and consideration for the principles of confidentiality may lead to HIV-infected people to lose confidence; for fear that their status could be disclosed to others. This will impair the opportunities of positive relationship between physician and patient $[4,5]$.

However, there may be conflicts when a person discloses their HIV positive status to a counselor, but refuses to disclose this aspect to sexual partner. In this case, we have the classic situation where two ethical principles come into conflict. It is recommended to be the first client autonomy and preserve the privacy or violate privacy in order to avoid potentially fatal harm to an infected partner. On the one hand, the principle of autonomy requires confidentiality and prohibits disclosure of information to another person. On the other hand, the principles of benefit and harm as good and worse do not require that the life partner to be protected, giving the information required to avoid infection $[1,4,5]$.

Need to share information may be a requirement in this specific case. In long-term information process sharing means, the confidential information could not be limited to the physician or counselor, which means that prospective patients or clients would not share such sensitive information with their physicians and counselor. In this case, respecting the duty of not to harm could have dangerous consequences in the long term $[1,4,5]$.

Both principles - that of autonomy and the beneficence - must be carefully weighed and each case should be treated with extreme carefulness of ethical judgment $[1,4]$. The physician or counselor may violate confidentiality, strictly on a need to inform and to avoid possible harm if not done so.

\section{Condom use}

From a medical point of view, the condom is a medical device that prevents the exchange of fluids during sexual act. While the effectiveness raises technical problems, the condom is not specific ethical problems, but they are taking into account when we speak about the effects of its use, or when promote use $[2,4]$.

Some consider that promoting condom use may increase promiscuous sexual behavior, while others considered that sexual behavior is largely determined by other factors, but in order to prevent the consequences of this undesirable behavior, its use reduce the transmission of sexual diseases. Implications arising from the results of the studies about the effects of various factors on human behavior are influenced by the principles of applied ethics. A conflict between protection of the moral integrity of people, by reducing incentives for sexual promiscuity, and the wish to protect other people's health, through averting potentially fatal infections, may occur [4].

The respect for autonomy would help the selfpersonal determination [1]. According to this view, even if condoms have negative effects, information should not be banned so that people can decide its own. Studies have shown that promoting the use of condoms with different sexual partners it may increase the risk of transmission of HIV, even the risk of TB infection by contaminated air in some geographical areas where TB is burden and/or in crowded settings. In this case, the principles of beneficence and no harm require access to condoms. If studies show that promoting condoms could reduce the risk of transmission of HIV the same principles would have a moral obligation to save lives by protecting people of acquiring infection. In addition, justice may require the implementation of the method of protection to all those who are living in society, not only for those who are capable to pay $[1,4]$.

The purpose of trying to reduce transmission of HIV is changing the behavior and social conditions. All available opportunities should be presented to the people involved and every effort should be made to help people make decisions for the life based on the options available in accordance with current information and experiences. To evaluate the various claims and ethical principles to determine the best solution when ethical principles are in conflict, ethical decisions must be better informed [4].

Needle exchange for intravenous drug users

Supply of sterile needles and syringes to those who need these things because they are addicted by injectable drugs is a method of preventing HIV infection. Considered purely a technical issue, it poses no ethical problems, but these problems occurs when we take into consideration and promote the 
use of such devices. Because some drugs and use of heroin are harmful and illegal in many countries and some companies try to discourage their use, in order to obtain the means of safe injecting, a drug may become an ethical problem [4].

Answers to these questions depend on the results of the studies. Without doubt that supplies sterile needles will remove the risk of HIV transmission to those who use drugs intravenously. But studies have since shown that the introduction of schemes of those changes will not increase or decrease the use of these drugs. If availability does not modify significantly the behavior of using drugs, it will be a strong moral obligation to provide these devices to save lives $[1,6]$. The best way to reduce transmission of HIV by those who are already infected is primary and secondary prevention of drug use and abuse [4].

\section{Informed consent and forced testing}

Informed consent is based on the respect for autonomy which requires that no patients shouldn't be forced to perform any therapeutic or diagnostic procedures that may affect their health and/or their future life, without previous adequate and complete information. This should enable them to take informed and independently decisions on their future $[4,6]$.

Explicit consent is not required for all laboratory routine investigations, which do not presents particular risks and that are necessary for treatment, in this case only general consent of the patient is enough. HIV test is different, not only because the infection is still incurable, but also because of the personal, social and economic consequences, for these people, in particular stigma and discrimination when HIV diagnosis is made public.

Therefore, HIV screening testing of TB patients should be done only if it obtains informed consent of the person concerned, without any form of coercion or persuasion. This implies that forced testing, for whatever reason, is not ethical (including testing for employment, education access, entry into the country, or medical treatment).

We can create a conflict between the rights of HIV infected individuals, TB patients and human society that wants to protect a large number of its members through the control mechanisms that restrict the individual rights. Like in some past epidemics, utility arguments can be used to justify the elimination of individual rights to informed consent and confidentiality in favor of the majority. However, in practice these extreme measures are neither necessary nor useful for HIV; the best way of prevention is providing information, seeking voluntary cooperation, and no forced testing $[5,7,8]$.

\section{Research}

There are several ethical issues related to research in HIV/AIDS with or without related TB. These concern, for example, to research on human subjects in the development of new medicines, the access to experimental medicines by sick and desperate patients, and ethical principles for leadership development of vaccines and clinical trials $[4,9]$. There are international codes and principles for regulating the ethical in all preliminary studies involving ethical human subjects. Nuremberg Code (1947), Declaration of Helsinki of the World Medical Association (1975) and the Council for International Organizations of Medical Sciences (CIOMS) (1990) - protects the rights of all those taking part in any study about new treatments or vaccines. These codes clearly specifies that people are invited to participate voluntarily in studies after giving their informed consent, and if they are exposed to minimal risks $[4,10]$.

Studies related to TB and HIV/AIDS are no exception, but ethical issues arise when the study is the only way in which ill desperate patients can hope to receive any potentially effective treatment. The principle of beneficence urges that more and more such patients to participate in the trials. But the principle of non-harm it forces researchers to carefully and gradually increase the number of those receiving the experimental therapy, as evidence of its efficacy and lack of side effects gained. This would conflict with the autonomy of patients who want them to decide whether to assume the risk of side effects of a possible beneficial therapy $[9,11]$.

An ethical issue is increasing in HIV vaccine studies. When research into the effectiveness of an experimental vaccine requires subjects to be in a continuous risk, if studies show its effectiveness, ethical issues are composed by the opportunity of participants to create a false feeling of security and 
that risk can be reduced through health education. In addition to these concerns of non-malefic there are worry about justice that occur when those recruited for such studies are too poor or too sick to be advised not to participate. Justice requires that both risks and benefits of development, production and distribution of potential therapies should be shared globally, without harm to vulnerable groups or countries [9].

\section{Allocation of resources}

Proper apportionment of resources is a major assumption of adequate care for people living with HIV/AIDS and effective prevention of transmission of HIV and TB infection. This applies to all social and economic levels. At a level of community personal, financial, emotional and spiritual resources must be mobilized to achieve full participation of people living with AIDS in communal life, and to give them care which is necessary for their physical and emotional well-being. At a national level, HIV/AIDS associated with TB must receive the full attention of leaders and their support in mobilizing necessary resources to this issue, both in relation to human suffering involved, and the social and economic consequences of the co-infection and implications of both diseases on national level.

At a global level, the international medical community must ensure that adequate measures are taken to fight against the global HIV pandemic, the co-infection HIV-TB which affects all regions and continents. Until now, resources for treatment and care of patients with AIDS and for prevention of HIV transmission were unevenly divided. Although more than $80 \%$ of HIV infections occur in poorer countries, they receive only a small part of the resources consumed at international level for HIV/AIDS [12].

This raises serious problems of distributive justice. Justice requires the greatest care for those with the greatest needs. This means in practice, that resource should be redistributed, giving each country a fair part, allowing these countries to establish programs tailored to the local situation. The purpose of this process - to reduce both the burden of those directly affected and the further spread of the infection - is in accordance with the common good of humanity.

\section{Debt of health professionals}

Unfortunately there are reports of people living with HIV/AIDS and TB whom were forbidden entry to some health institutions and churches, who's care was refused by health professionals although these people were sent for treatment, help or medical advice. In terms of ethics, these attitudes are not justified. Access to care is a right for all, including those infected with HIV and TB. There is no medical or ethical reason to restrict this right [4].

Some health professionals have referred to the increased risk of contracting HIV and TB of those who are involved in treating HIV positive patients and/or TB patients. This is not justified by the studies performed so far in relation to the real exposure risk of health professionals. So far very few health professionals are HIV positive and even less with TB disease and it is difficult to demonstrate that they have got the infection through actions related to professional activities. In general, health professionals are not considered as having a higher risk of HIV or TB infection compared to the rest of the population.

Taking the necessary precautions, the risk of acquiring occupational infection is very low. Statistically, a needle prick of blood infected with HIV will lead to an infection in $0.3 \%$ of cases $[13,14]$. International bodies governing professional's activity, concerning treatment of HIV infected people considered that they should be treated like other patients and healthcare workers who refuse to give treatment will be considered at a major breach of professional conduct [15]. In outbreaks of TB among hospital personnel, infection may appear but disease is rare. There were several cases with MDR-TB and nosocomial transmission of drug sensitive disease in health care workers $[16,17]$. This view is supported by all four ethical principles [1]. A sustained education of healthcare workers about HIV-TB infection methods of control should be conducted. 


\section{Conclusions}

1. Appearance of HIV/AIDS and related TB has resulted in changing ethical approach in modern medicine; some ethical issues have acquired a new relevance such as the ethics of relations between staff and patients, or ethics of interprofessionals relations, and some issues in launching health policies.

2. Preserving privacy in HIV is essential to establish a durable relationship in time, the trust between doctor/counsellor/psychologist and patient. However sometimes doctor/ counsellor/ psychologist may violate confidentiality strictly on a need to inform and to avoid possible harm.

3. Given that HIV testing to all TB patients as a routine test and diagnosis of HIV infection can produce major changes in way of life of the person concerned is important that testing be preceded by counseling and to achieve agreement with that person.

4. Even if the use of condoms has negative effects on the moral, necessary information should not be prohibited, so that people can decide on its own precautions. The longer a person is better informed so can take a competent decision to her life.

5. Because drug use and heroin is a particularly harmful and illegal in many countries changing the syringes used by intravenous drug users can be an ethically problem. Needle change is the only one of the ways to reduce one of the many risks and threats of people who uses drugs.

6. Ethical issues in trials that take place in HIV/AIDS and TB patients occur when desperate ill patients may benefit from treatment available only in clinical trials, and there is no clear evidence on the effectiveness of therapy without fully knows the side effects. Ethical issues arise when investigating the efficacy of a vaccine, because patients are exposed to a continuous risk of contracting the disease, and by creating a false feeling of security.

7. Both at community and national and global HIV/AIDS and TB must ensure a fair distribution of resources, so that most care is given to those with the greatest needs. Although more than $80 \%$ of HIV-TB co-infection occur in poorer countries, they receive only a small part of the resources consumed in international HIV/AIDS and $\mathrm{TB}$, therefore at this time create serious problems related to the right distribution.

8. Access to medical care is a right of all patients, including those infected with HIV and there is no medical or ethical reason to restrict this right. Because the risk of acquisition of HIV is very small professional bodies which regulate the conduct of international business have requested that people infected with HIV be treated like other patients, and refusal of treatment will be considered a major breach of professional conduct.

\section{References:}

1. Beauchamp, T. \& Childress, J. (1994). Principles of Biomedical Ethics (4th ed.), Oxford. Oxford Univ. Press.

2. Henderson, D.K. et al. (1990). Risk for Occupational Transmission of HIV-1 Associated with Clinical Exposures, Ann. Int. Med., 113, $740-46$.

3. Reamer, F. (1991). AIDS: The Relevance of Ethics, in Reamer F. (Eds.), AIDS and Ethics, (pp. 1-25), New York: Columbia Univ. Press.

4. Benn, C. \& Boyd, K.M. (1996). Ethics, medical ethics and HIV/AIDS. Ecumenical Review. 48(2), $222-232$

5. Harris, J. \& Holm, S. (1993). If Only AIDS Were Different, in Hastings Center Report, 23(6), 6-12.

6. Drane, J. (1992). AIDS and Ethics, in FuenzalidaPuelma, H., Linares Parada, A.M. et Serrano, LaVertu D. (Eds.), Ethics and Law in the Study of AIDS, Washington, D.C., Pan American Health Organization [PAHO], pp. 129-137.

7. Boyd, K.M. (1992). HIV Infection and AIDS: The Ethics of Medical Confidentiality, Journal of Medical Ethics, 18, 173-79.

8. Boyd, K.M. (1990). HIV Infection: The Ethics 
of Anonymised Testing and of Testing Pregnant Women, Journal of Medical Ethics. 16, 173 -178.

9. Connor, S.S. (1992). International Legal and Ethical Aspects of Developing and Distributing an HIV Vaccine, in Fuenzalida-Puelma, H., Linares Parada, A.M. et Serrano LaVertu, D. (Eds.), Ethics and Law in the Study of AIDS, Washington, D.C., Pan American Health Organization [PAHO], pp.162-169.

10. Council for International Organizations of Medical Sciences (CIOMS). (1991). Ethics and Epidemiology: International Guidelines. Geneva, CIOMS.

11. Boyd, K.M. (1992). AIDS, Ethics, and Clinical Trials, British Medical Journal, 305, 699-701.

12. Benn, C. (1994). Justice and AIDS, One World. 201, 6-8.
13. De Andres, R. et al. (1994). Update on EC Multicenter Study of Occupational Exposure to HIV in Health Care Workers, 10th International Conference on AIDS, Abstract 273 B/D, Yokohama.

14. Gillon, R. (1994). Principles of health Care Ethics, Chichester, John Wiley \& Sons.

15. Zuger, A. (1991). AIDS and the Obligations of Health Care Professionals, in Reamer F. (Eds.), AIDS and Ethics, (pp. 215-239), New York: Columbia Univ. Press.

16. Dooley, S.W., Villarino, M.E. \& Lawrence, M. (1992). Nosocomial transmission of tuberculosis in a hospital unit for HIV-infected patients, JAMA. 267, 2632- 2635.

17. Menzies, D., Fanning, A. \& Juan, G. (1995). Tuberculosis among health care workers, $N$ Engl J Med. 332, 92-98. 